\title{
Research experimental of hipbone arthroplasties using tensometric models
}

\author{
Horatiu Bulea ${ }^{1, *}$ \\ ${ }^{1}$ Transilvania University of Brasov, Department of Manufacturing Engineering, Mihai Viteazu No.5, \\ Braşov, Romania
}

\begin{abstract}
Hip prostheses functionality is limited in time, inter alia, by mechanical damage to the femoral components (protrusion, limestone resorption femoral loosening), and by of accurate positioning of the piece femoral. To assess the importance of accurate positioning of the piece femoral hip arthroplasties we performed a tensometric study on experimental models on femurs dehydrated and lubricated, the prostheses were implanted cemented in varum, normal and valgus position. The specific model distortions were determined by applying tensometric stance on the external and internal cortical in maximal stress positions and request using static strength values similar to those that require bone during normal gait. Aim of this experiment is to determine the maximum load areas femur which has been implanted partial prostheses.
\end{abstract}

\section{Introduction}

Hip replacement is a surgical procedure in which the hip joint is replaced by a prosthetic implant. Hip replacement surgery can be performed as a total replacement or a hemi (half) replacement. Such joint replacement orthopaedic surgery is generally conducted to relieve arthritis pain or in some hip fractures. A total hip replacement (total hip arthroplasty) consists of replacing both the acetabulum and the femoral head while hemi arthroplasty generally only replaces the femoral head.

Hip replacement is currently the most common orthopaedic operation, though patient satisfaction short and long-term varies widely. In a partial hip replacement also called a hemi arthroplasty, only the femoral head is replaced and the acetabulum is left in place [1]. A partial hip replacement is done mainly to repair fractured hips.

The prosthetic device typically used in partial hip replacements incorporates a one-piece solid metal ball and stem that replaces the femoral head and upper femur, unlike the twopiece stem and ball design used in total hip replacements [2].

The Austin Moore and Thompson prostheses are common examples of the one-piece design used in partial hip replacements. Other prosthetic devices are considered modular and allow for different combinations of stem, neck length, and head size [3].

Improper placement of the prosthesis and the resulting biomechanical disturbances within the hip joint (excessive elongation or shortening of the extremity or improper

\footnotetext{
*Corresponding author: bulea@unitbv.ro
} 
rotation of the implant) are responsible for failure of hemiarthroplasty [4]. Inadequate $p$ calcar seating, insufficient residual femoral neck length, insufficient metaphyseal fill, and errors in sizing the prosthesis are all associated with early failure of the hemiarthroplasty [5].

Because of technical possibility during the operation, (shape, size and position of the bone) prosthesis can be implanted always in normal position, resulting varum and valgus positions. The objective of the present study is to analyze and evaluate the importance of a correct femoral part positioning in arthroplasties.

\section{The implant condition of the prosthesis and experimental methods}

\subsection{The implant condition of the prosthesis}

Hip prosthesis functionality is time-limited, in part by the femoral piece mechanical impairment (protrusion, femoral calcar resorption, de cementation).

In order to evaluate the importance of a correct femoral part positioning in arthroplasties, we performed a tensometric study on experimental models: dehydrated and degreased thighbones in which Moore prostheses were implanted and cemented in varum, normal and valgus position.Fig.1 designed approximately similar to anatomical shape of femoral head size: $45 \mathrm{~mm}$.

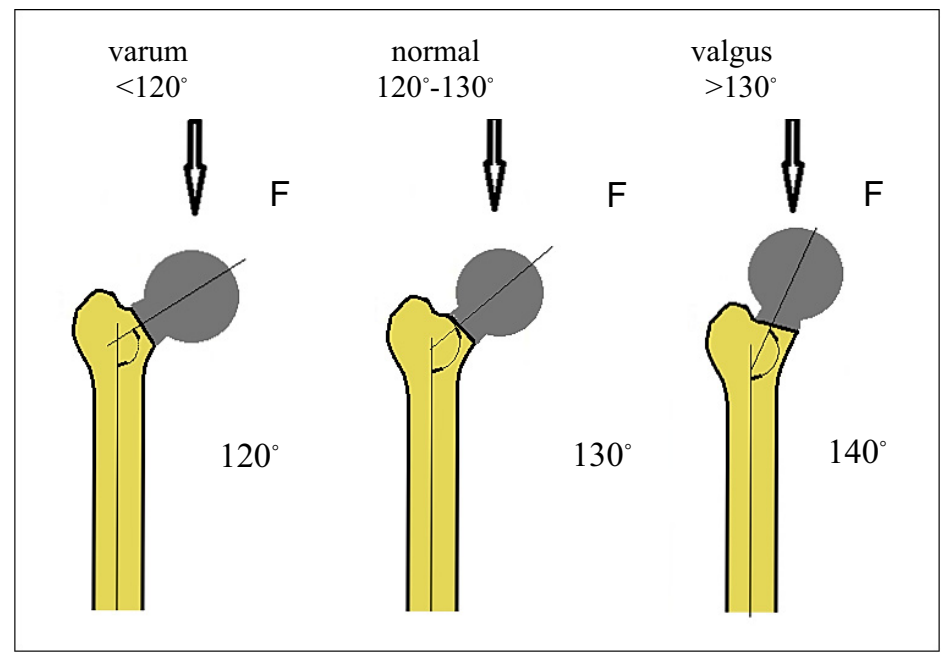

Fig. 1. Forces apply on spherical head of prostheses implanted and cemented in varum, normal and valgus position.

\subsection{The experimental methods}

The specific model distortions were determined by applying different forces on spherical head of prostheses in conditions similar to real ones they support bone in the human body and measurement with strain gauges deformations that occur on the surface of bone in areas highlighted on the external and internal cortical in maximal stress positions Fig. 2 and the experimental bone Fig. 3 . 
It is known that Young's modulus in bones has different values according to individual age and measurement area. Specific distortions were therefore determined only in points where tensometric stamps had been applied.

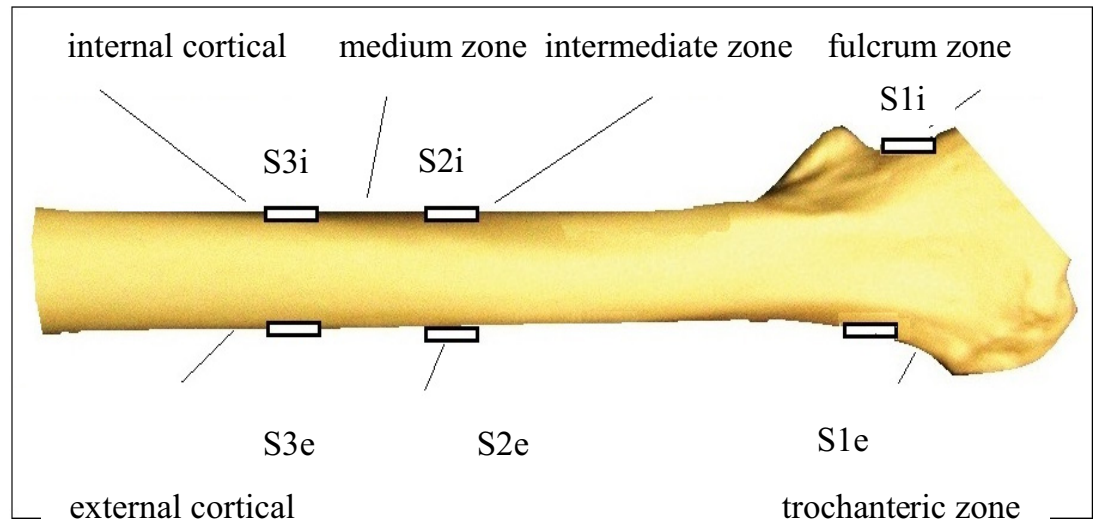

Fig. 2. Tensometric stamps highlighted on the external and internal cortical.

To conduct this experiment we are using the apparatus of Fig. 4.

The specific distortions Ds $[\mathrm{mA}]$ was measured for each tensometric stamp ten times for each of the five driving forces $\mathrm{F}_{1}=250[\mathrm{~N}], \mathrm{F}_{2}=300[\mathrm{~N}], \mathrm{F}_{3}=350[\mathrm{~N}], \mathrm{F}_{4}=400[\mathrm{~N}], \mathrm{F}_{5}$ $=450[\mathrm{~N}]$, for each positions of prostheses: varum, normal and valgus, the values considered are the average of measured values, the results are presented in Table. 1 Forces have been developed by means of a mechanic dynamometer, calibrated in the physics laboratory of the Transylvania University.

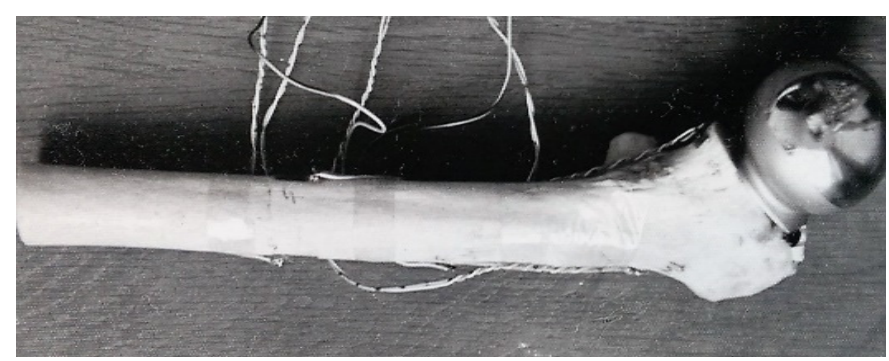

Fig. 3. Experimental bone with prostheses .

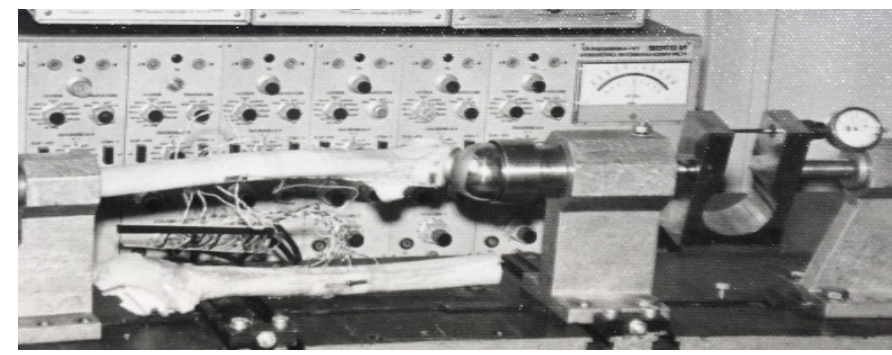

Fig. 4. Experimental installation.

Because the modulus of elasticity of bone material shows different values depending on the age of subject, measuring deformations were determined only specific points of placement of strain gauges. On external cortical they were obtained tensile stresses and compression on the internal cortical. 
Table 1. Mean values of the Ds deviation unit in $\mathrm{mA}$.

\begin{tabular}{|c|c|c|c|c|c|c|c|}
\hline \multirow{3}{*}{$\begin{array}{c}\mathbf{F} \\
{[\mathbf{N}]}\end{array}$} & \multirow{3}{*}{$\begin{array}{l}\text { Tenso } \\
\text { metric } \\
\text { stamp }\end{array}$} & \multicolumn{6}{|c|}{$\mathbf{D s}[\mathbf{m A}]$} \\
\hline & & \multicolumn{2}{|c|}{ Varum } & \multicolumn{2}{|c|}{ Normal } & \multicolumn{2}{|c|}{ Valgus } \\
\hline & & internal & external & internal & external & internal & external \\
\hline 250 & 1 & 101 & 130 & 81 & 120 & 74 & 112 \\
\hline 300 & 1 & 109 & 134 & 86 & 127 & 79 & 118 \\
\hline 350 & 1 & 116 & 143 & 91 & 132 & 84 & 123 \\
\hline 400 & 1 & 125 & 158 & 98 & 139 & 91 & 131 \\
\hline 450 & 1 & 133 & 166 & 105 & 146 & 97 & 139 \\
\hline 250 & 2 & 56 & 109 & 53 & 90 & 49 & 82 \\
\hline 300 & 2 & 67 & 116 & 64 & 97 & 54 & 91 \\
\hline 350 & 2 & 73 & 121 & 71 & 103 & 61 & 98 \\
\hline 400 & 2 & 81 & 133 & 79 & 111 & 68 & 106 \\
\hline 450 & 2 & 89 & 143 & 85 & 126 & 74 & 118 \\
\hline 250 & 3 & 105 & 193 & 92 & 180 & 97 & 188 \\
\hline 300 & 3 & 111 & 201 & 101 & 189 & 105 & 196 \\
\hline 350 & 3 & 121 & 216 & 109 & 198 & 113 & 205 \\
\hline 400 & 3 & 129 & 228 & 115 & 207 & 119 & 216 \\
\hline 450 & 3 & 138 & 239 & 123 & 216 & 127 & 224 \\
\hline
\end{tabular}

The variation of Ds $[\mathrm{mA}]$ parameter in the conditions presented above, are presented in Fig. 5 for tensometric stamps $\mathrm{S} 1_{\mathrm{i}}$ on fulcrum zone and in Fig. 6 for tensometric stamps $\mathrm{S} 1_{\mathrm{e}}$ on trochanteric zone in varum, normal and valgus position of implanted prosthesis.

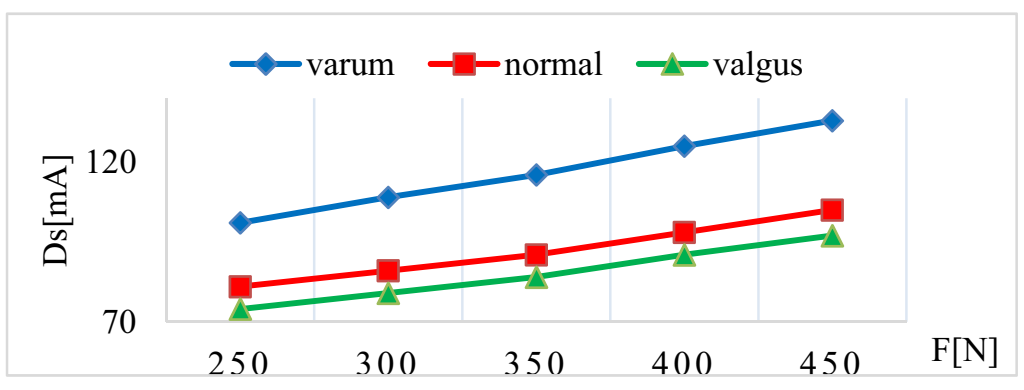

Fig. 5. The dependence of specific distortions $\operatorname{Ds}[\mathrm{mA}]$ from variation of force request $F[\mathrm{~N}]$ for tensometric stamps $\mathrm{S} 1_{i}$ on fulcrum zone. 


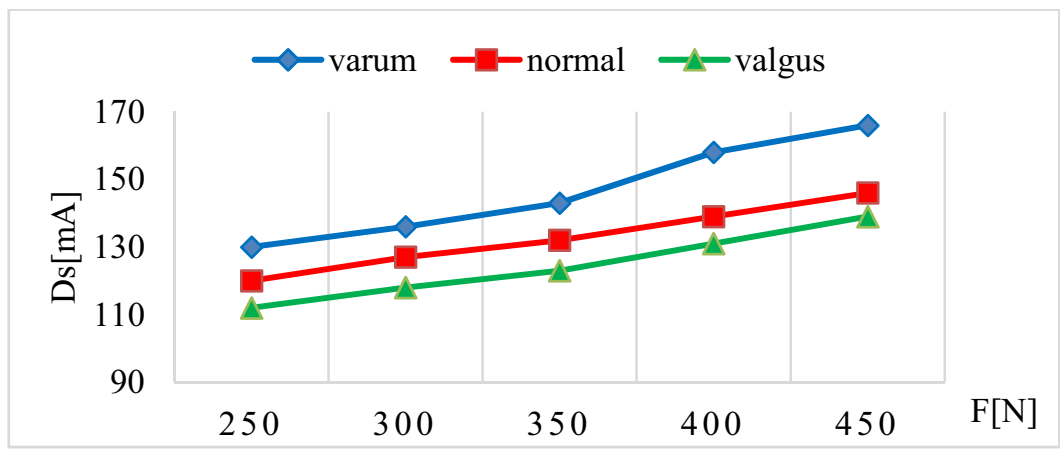

Fig. 6. The dependence of specific distortions $\mathrm{Ds}[\mathrm{mA}]$ from variation of force request $\mathrm{F}[\mathrm{N}]$ for tensometric stamps $\mathrm{S} 1_{\mathrm{e}}$ on trochanteric zone.

The variation of Ds[mA] parameter in the conditions presented above, are presented in Fig. 7 for tensometric stamps $S 2_{i}$ on internal cortical in intermediate zone and in Fig. 8 for tensometric stamps $\mathrm{S} 2_{\mathrm{e}}$ on external cortical in intermediate zone in varum, normal and valgus position of implanted prosthesis.

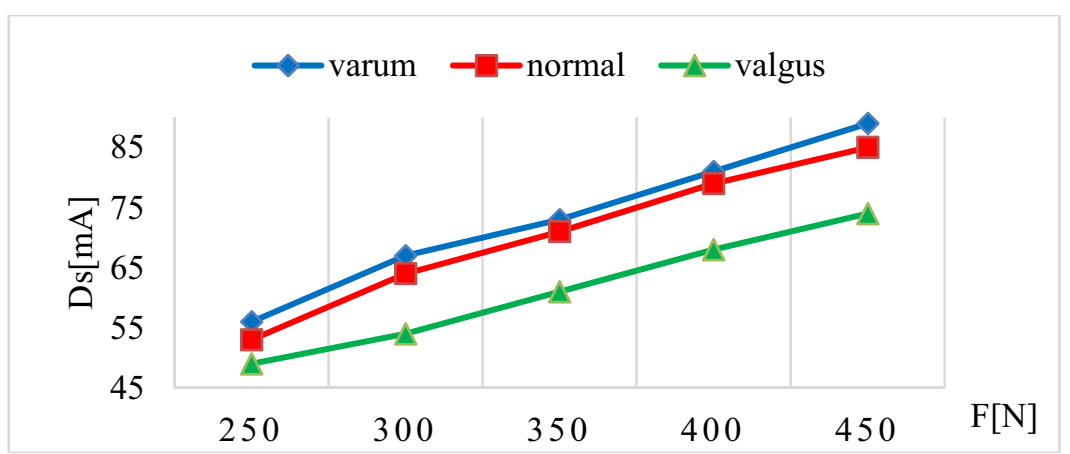

Fig. 7. The dependence of specific distortions $\operatorname{Ds}[\mathrm{mA}]$ from variation of force request $F[\mathrm{~N}]$ for tensometric stamps $\mathrm{S} 2_{\mathrm{i}}$ on internal cortical.

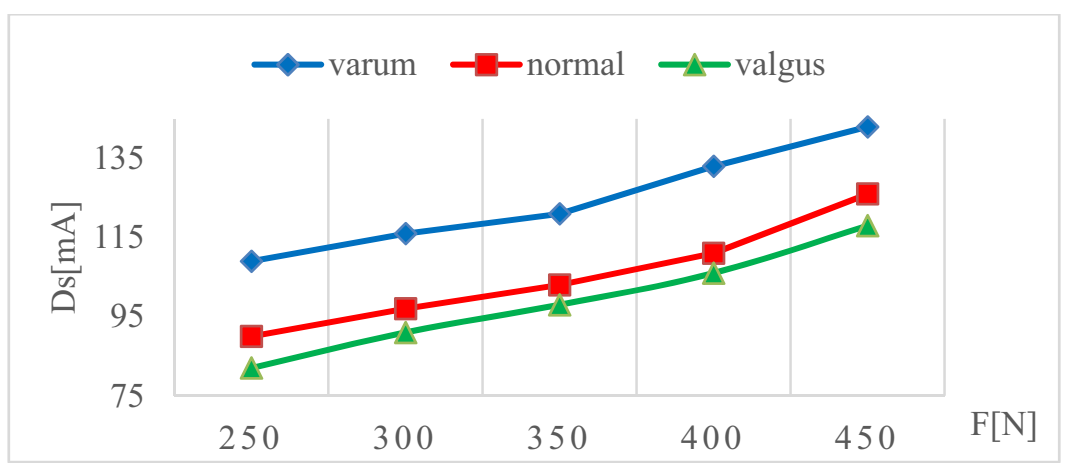

Fig. 8. The dependence of specific distortions $\mathrm{Ds}[\mathrm{mA}]$ from variation of force request $\mathrm{F}[\mathrm{N}]$ for tensometric stamps $\mathrm{S} 2 \mathrm{e}$ on external cortical.

The variation of Ds[mA] parameter in the conditions presented above, are presented in Fig. 9 for tensometric stamps $\mathrm{S} 3_{\mathrm{i}}$ on internal cortical $\mathrm{n}$ medium in varum, normal and valgus position of implanted prosthesis. 


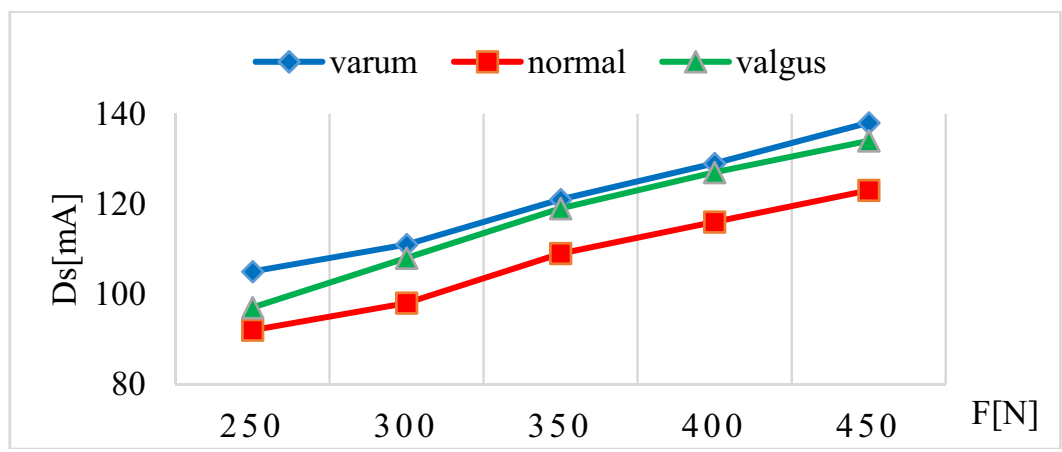

Fig. 9. The dependence of specific distortions $\mathrm{Ds}[\mathrm{mA}]$ from variation of force request $\mathrm{F}[\mathrm{N}]$ for tensometric stamps $\mathrm{S} 3 \mathrm{i}$ on internal cortical.

The variation of $\mathrm{Ds}[\mathrm{mA}]$ parameter in the conditions presented above, are presented in Fig. 10 for tensometric stamps $\mathrm{S} 3_{\mathrm{e}}$ on external cortical medium zone in varum, normal and valgus position of implanted prosthesis.

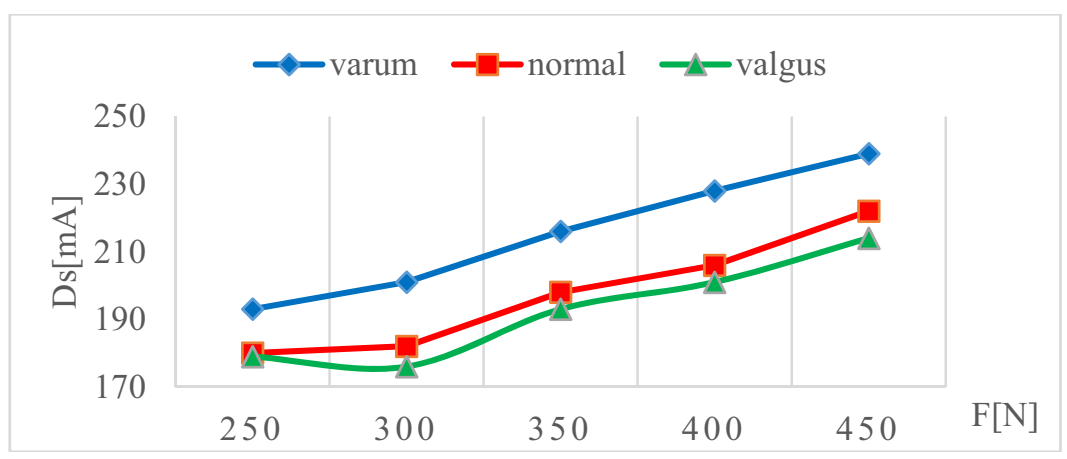

Fig. 10. The dependence of specific distortions $D s[\mathrm{~mA}]$ from variation of force request $F[\mathrm{~N}]$ for tensometric stamps $\mathrm{S}_{\mathrm{e}}$ on external cortical.

The variation of Ds[mA] parameter in the conditions presented above, are presented in Fig. 11 for tensometric stamps $\mathrm{S}_{1}$ on fulcrum zone and for tensometric stamps $\mathrm{S} 1_{\mathrm{e}}$ on trochanteric zone in varum, position of implanted prosthesis.

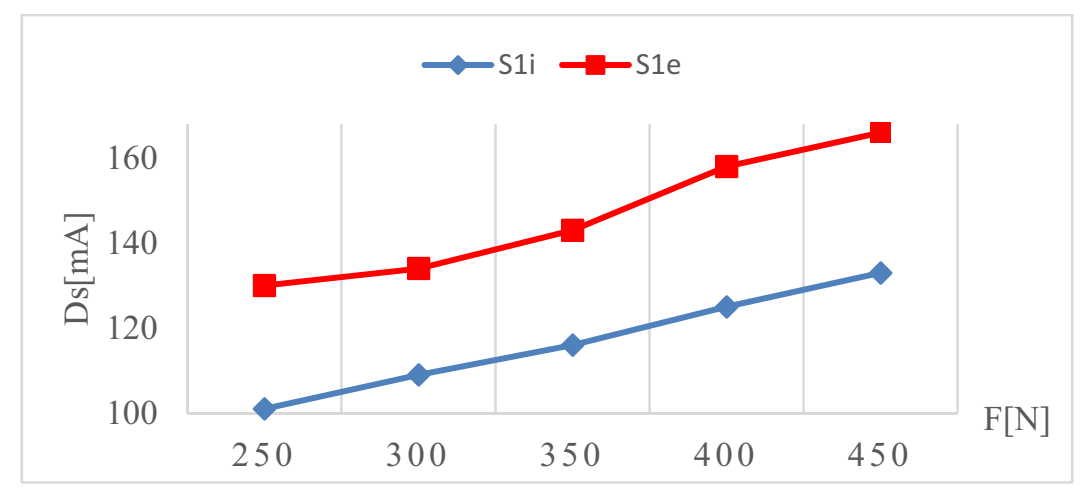

Fig. 11. The dependence of specific distortions $D s[\mathrm{~mA}]$ from variation of force request $F[\mathrm{~N}]$ for tensometric stamps $\mathrm{S} 1_{\mathrm{i}}, \mathrm{S} 1_{\mathrm{e}}$ in varum, position of implanted prosthesis. 
The variation of $\mathrm{Ds}[\mathrm{mA}]$ parameter in the conditions presented above, are presented in Fig. 12 for tensometric stamps on intermediate zone $\mathrm{S} 2 \mathrm{i}$, for internal cortical and $\mathrm{S} 2 \mathrm{e}$ on external cortical for normal position of implanted prosthesis.

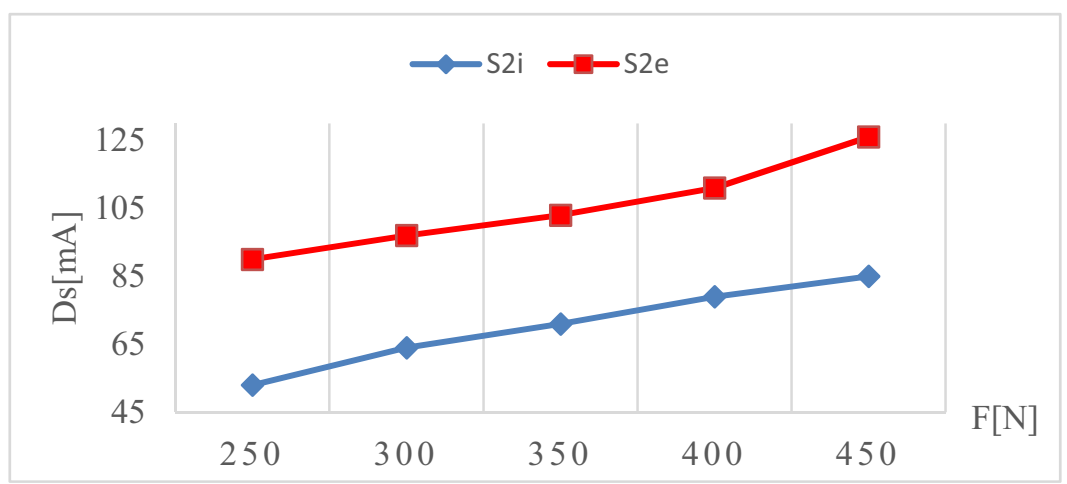

Fig. 12. The dependence of specific distortions Ds[mA] from variation of force request $F[N]$ on intermediate zone for tensometric stamps $\mathrm{S} 2 \mathrm{i}, \mathrm{S} 2 \mathrm{e}$ for normal position of implanted prosthesis.

The variation of Ds[mA] parameter in the conditions presented above, are presented in Fig. 13 for tensometric stamps on medium zone $\mathrm{S} 3 \mathrm{i}$, for internal cortical and $\mathrm{S} 3_{\mathrm{e}}$ on external cortical for valgus position of implanted prosthesis.

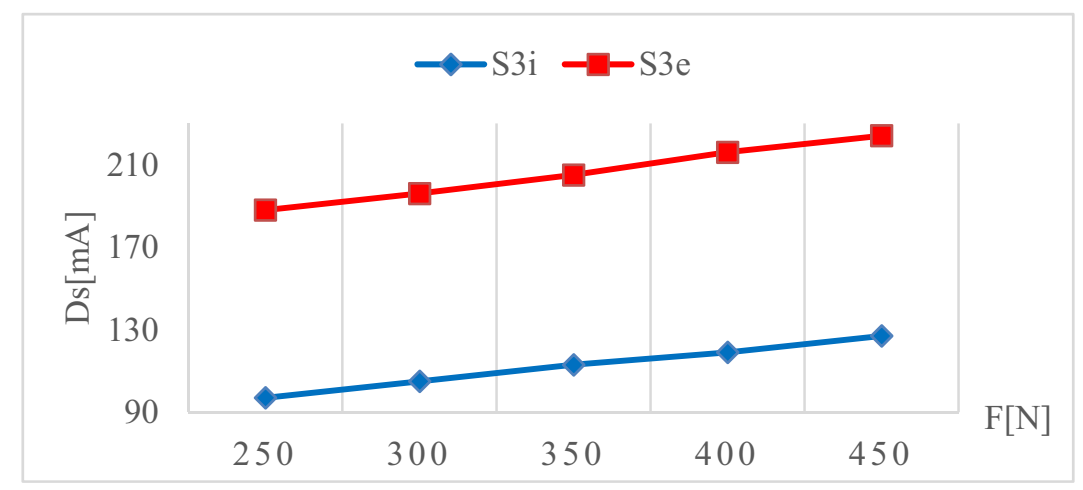

Fig. 13. The dependence of specific distortions $\mathrm{Ds}[\mathrm{mA}]$ from variation of force request $\mathrm{F}[\mathrm{N}]$ on medium zone for tensometric stamps $\mathrm{S} 3_{\mathrm{i}}, \mathrm{S}_{\mathrm{e}}$ for valgus position of implanted prosthesis.

\section{Conclusions}

Ascending the analysis results presented in tables and above graphs the following conclusions:

1. Analyzing the experimental parameters, important influence of deformations of the bone are: first the size of the force apply on spherical head of prostheses, and second the position in which it was implanted prostheses.

2. For same forces apply on spherical head of prostheses deformation being significant higher in varum position of prostheses.

3. For same forces apply on spherical head of prostheses deformation is significant higher in middle zone of bone tail end of prostheses.

4 .The cortical external requests are on cortical tension and on internal compression.

5. For same forces apply on spherical head of prostheses deformation is higher for external cortical that internal cortical in all zone of bone. 
6. With increasing strain force deformation increases more in varum position of the prosthesis for all zone.

7. Stress diagrams were set out for the specific distortions they showed an almost linear variation within the limits of the charges applied, thus proving that bones submit to Hooke's law.

8. The most important conclusion resulting from the experiments is that deformations are real, they lead to a request extra bone in the contact areas of the prosthesis.

\section{References}

1. V. Meulen, M.C.H. Allen, W.A. Giddings, V.L. Athanasiou, K.A. Poser, R.D. Goodman, S.B. Smith, R.L. Beaupré, Effect of hemi arthroplasty on acetabular cartilage, Project Reports. VA Palo Alto Health Care System's Bone and Joint Rehabilitation Research and Development Center (1996)

2. A. Mecke, I. Lee, J.R. Baker jr., M.M. Banaszak Holl, B.G. Orr, Eur. Phys. J. E 14, 7 (2004)

3. ${ }^{* *}$ Coding for Hip Replacement Surgery, AHIMA, 25/13, 26 (2013)

4. P. Bhosale, A.V. Suryawanshi, Total hip arthroplasty for failed aseptic Austin Moore prosthesisand, Amber Mittal Indian J Orthop. 46, 297-303 (2012)

5. P. Weinrauch, Intra-operative error during Austin Moore hemiarthroplasty, J Orthop Surg 14, 249 (2006) 